\title{
Recent Research and Prospect on Feature Recognition of Three-dimensional Model
}

\author{
Yangdong Shi, Zhaohui Deng and Junwu Zhong \\ Hunan Provincial Key Laboratory of High Efficiency and Precision Machining of Difficult-to-Cut Material, Hunan University of \\ Science and Technology, Xiang Tan, 411201, China \\ Intelligent manufacturing institute of HNUST, Xiang Tan, 411201, China
}

\begin{abstract}
A feature recognition technique is the intelligent interface of CAD/CAPP. Through extracting and recognizing the tolerance and surface roughness, the design features such asholes, steps and other design features in a 3D model of parts, the CAD/CAPP can be combined efficiently, and then the CAD/CAPP/CAM system can be integrated. This technique also helps to shorten the cycle of product development, especially process preparation, and reduce the design cost. In this paper, the 3D model features recognition approaches about boundary pattern matching, volume composition, manufacturing resources, and hybridization method are reviewed, and compared in terms of identifying efficiency, identifying accuracy, identifying flexibility, machinability of features, and utilization of manufacturing resource information in a process environment is conducted on the 3D model feature recognition approaches. The main issues of machining feature recognition of the part model are also discussed, and the direction of future researches in this field is presented. It is pointed out that the development of an efficient, accurate, flexible, automatic and intelligent machining feature recognition system will become an important research topic in the field of computer integrated manufacturing in the future.
\end{abstract}

Keywords-Three-dimensional model; Feature recognition; CAD/CAPP/CAM; Machining feature recognition based on boundary pattern matching; Machining feature recognition based on volume decomposition; Machining feature recognition based on manufacturing resources; Hybrid recognition of Machining Feature

\section{INTRODUCTION}

With the introduction of Industry 4.0, the industry has become ever more eager to look forward to intelligent workshops and smart factories, and has raised increasingly high demands on CAD/CAPP/CAM automation and integration. However, CAD/CAPP/CAM data lack interactivity and patency, and there are heterogeneity in expression patterns [1]. CAD/CAPP/CAM systems are not compatible with each other. Structural design data and manufacturing process data can be not fully correlated, coordinated and integrated, and the degree of automation is not distinguished. In addition, the traditional process design mode requires the craftsman to input a large amount of manufacturing process information in the CAPP system, and the information loss and distortion problems are serious. After the design model is changed, the craftsman needs to remodel. This not only makes the craftsman fall into non-designal repetitive work, but also the consistency and integrity of the information is difficult to guarantee. 3D model processing feature recognition technology can shorten the process preparation period of parts, make the CAD/CAPP system effectively connect, and realize the automation and integration of CAD/CAPP/CAM, breaking the CAD/CAPP/CAM "information island" obstacle. Therefore, it is extremely important to identify the processing features of the 3D model.

The research of feature recognition has been pursued for many years as a key link between computer-aided design (CAD) and computer-aided manufacturing (CAM) [2]. Over the past decades, a lot of researches on this feature recognition technology have been performed at home and abroad, and great progress has been made. At present, the processing feature recognition methods are mainly divided into the following categories: The first type of method performs pattern matching based on the boundary conditions [3], such as based on the graph [4], based on the trace [5], based on the rule [6], based on the neural network [7], and so on. The second type of method is based on volume decomposition. The third type of method is based on the manufacturing resource mapping. The fourth category is a hybrid approach. The rule-based approach was proposed in the late 1970s to better identify simple independent features, but it is less efficient when deriving complex features. In the mid-1980s, graph-based methods gained widespread attention at home and abroad, but there were things in the identification of intersecting features. In response to this problem, methods based on traces and volume decomposition have been put forward at home and abroad. In the mid-1990s, domestic and foreign scholars began to introduce neural network methods in feature recognition technology. The idea of Manufacturing resources, the method of processing surface-manufacturing resource mapping has gradually received attention. The hybrid method integrates the advantages of various methods and has gradually been part of the trend and tendencies in the development of processing feature recognition technology. In the 21st century, process feature recognition technology entered the application stage, and various process software and process feature recognition systems were continuously developed. The CAPP system is promoted by Western Boeing. Lockhead and other companies are of profound significance for the integration, sharing and collaboration of product data. The feature recognition system developed by Nasr can communicate with different CAD/CAM 
systems [8]. Marchetta developed a system that identifies manufacturing features [9]. In China, it has made a significant contribution to the identification of the processing features of composite structural parts such as aircraft structural parts, boxes and cylinders.

A product model is described by a number of surface elements and their relationships, whilst a machining sequence relates to the types and locations of machined features. A machined feature is defined as a sequence of surface elements which could be machined by a single tool, though not necessarily in one pass [10]. In the evaluation of processing feature recognition methods, recognition efficiency and accuracy have traditionally been the focus of scholars at home and abroad. At the same time, the method with accurate recognition flexibility has important practical significance in the identification of different complex parts. In addition, the processing feature recognition method must fully consider the processability of the identifiable features, improve the utilization rate of the manufacturing method information and process information, which is of profound significance for the processing and manufacturing of the part features. This paper mainly analyzes and discusses the development status and research status of 3D model processing feature recognition methods, from recognition efficiency, recognition accuracy, recognition flexibility, processability of identifiable features and utilization of manufacturing resource information in a process environment. In this paper, several processing feature recognition methods are compared. Finally, the research focus and development direction of processing feature recognition technology are emphasized.

\section{Three-Dimensional Model Processing Feature RECOGNITION METHOD}

\section{A. Rule-based Approaches}

The rule-based method uses the boundary rules of the same feature to formally describe and classify the features of the entity model, which has a good recognition effect on simple features. Some heuristic information as key elements and ordered searches, has been introduced into the rules so that control mechanism is able to make good use of human intelligence and experience to improve the search performance [10].

However, most methods extract the features from a part model based on a fixed set of recognition rules and the resulting feature data of the recognition process is not easily modified. It is means that the only channel of user interaction in the recognition process is obtained by encoding the user's preference in the recognition rules and letting the recognition rules take over the entire recognition process in a batch mode. It is indirect and inconvenient for user [11]. In addition, since all feature types cannot be defined and described by rules, the intersection feature cannot be identified, and there are great difficulties in the rule description and recognition of complex features, and there is a problem of low efficiency in the process of complex feature deduction.

Automatic recognition of features is difficult, and more difficult is the recognition of feature interaction. The obvious limitation of the rule-based methods is that they apply to a domain of specific features. Typically, for a rule-based system, predefined rules for recognizing such feature are not possible owing to the many possibilities of intersections. The difficulties of recognizing intersecting features arise from the fact that the intersection destroys part or all of the characteristics of basic features [12]. In order to identify complex intersecting features, this method is increasingly combined with other methods to develop into one of the important branches in the hybrid method, such as the use of heuristic rules for the accessibility analysis of the processing surface combined with the manufacturing feature trace method and annotation semantic information. The way to identify the fixed axis machining class intersection manufacturing characteristics.

\section{B. Graph-based Approaches}

The graph-based approach has evolved over the years and continues to be one of the mainstream methods to this day. This method extracts some boundary conditions of the 3D model of the edges and nodes of the adjacency graph, uses arc and additional information to represent the attribute information between the nodes, and finally matches the feature library.

The research results of this method are more. Joshi and Chang developed an algorithm for feature recognition based on an attributed adjacency graph(AAG) [13]. The features such as holes, steps and virtual pockets are recognized and then extracted from the solid model data structure by using predefined rules in the system. However, recognition of curved features, T-slots and different types of holes is not included in the algorithm. Most feature recognition methods dveloped so far are effective with non-intersecting features in polyhedral geometries and experience difficulties with a part model containing free-form surfaces or interactiong features, although some limited remedies have been suggested [11]. In the aspect of the attribute adjacency graph, The ability to recognize the need to be further improved. In terms of extended attribute adjacency graphs, Li expands the properties of feature seed faces and adjacent edges, and can identify common surface features and intersecting features in aircraft structures [14].Ou used the isomorphic features of the graphs to perform subgraph matching on the basis of the subgraphs, and can identify the features of the convex and concave features [15, 16]. Sunil proposed a method that can decompose more feature subgraphs [17]. These methods effectively increase the efficiency of subgraph matching. In the aspect of complex feature processing and recognition, Gao used the Delaunay method to triangulate the surface, and has a wholesome recognition effect on the three-dimensional model of the part containing the finely divided surface [18]. Da-Lei extended, intersected, and segmented feature plans to complete the separation of intersecting features [19].

The traditional graph-based approach has great advantages in identifying simple independent features, but there are deficiencies in identifying intersecting features. In recent years, domestic and foreign scholars have improved the customary attribute adjacency graphs, proposed some different types of attribute adjacency graphs, and further expanded their attribute information. Through a series of technical means, the graph-based method has made remarkable progress in subgraph matching efficiency, processing and recognition of complex 
features, and can identify general intersecting features and partial surface features. However, in general, it is still necessary to further improve its ability to recognize and process complex intersection features and complex surface features.

\section{Trace Based Approaches}

The trace-based method uses heuristic information such as topological geometric information of the feature to perform reasoning. The implementation process is shown in Figure I.

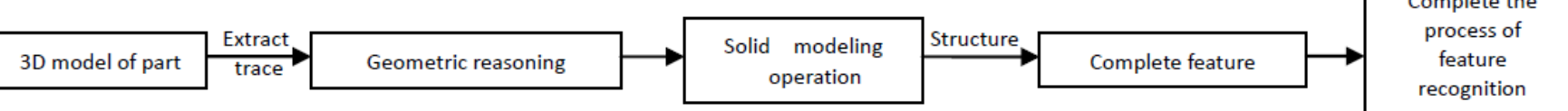

FIGURE I. TRACE-BASED PROCESSING FEATURE RECOGNITION METHODS.

By identifying and combining them, we are able to identify the topological features of the aircraft structural parts and the accuracy is high. Li proposed a method for reusable trace shape [20]. By detecting the inclusion, fusion and cross-correlation of general shape features, it is possible to identify common shape features of composite parts. The trace-based method can identify shared surface and intersecting features, and solves the problems of traditional graph-based methods in intersecting feature recognition to some extent. Selecting appropriate feature traces is essential to the trace method.

\section{Neural Networks Based Approaches}

The neural network-based method has the ability of fuzzy recognition and instance learning, which have high recognition efficiency and can recognize general intersecting features and incomplete features. The implementation process is illustrated in Fig. II. Yong used the continuity of normal vector and curvature to detect the data boundary points, selected the feature area or the block surface through human-computer interaction, and encoded the feature cross-sectional shape, section concavity and opening and closing property and its input neural network is trained [21]. Tan encodes the feature's top view shape and input it into the BP neural network, and finally completes the feature recognition process, but the human-computer interaction is too much [22]. How to encode the geometric topology information of the feature and better play the learning ability of the neural network is the future research direction based on the neural network method.

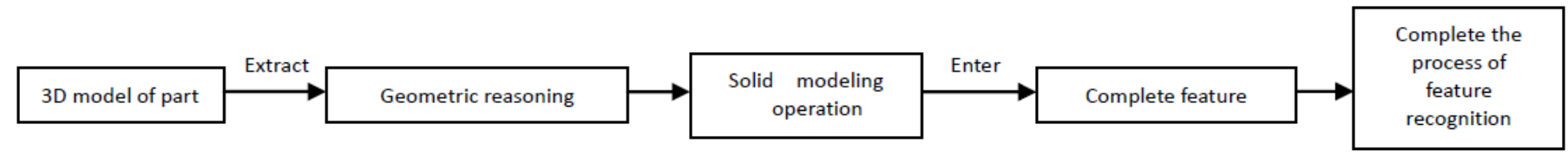

FIGURE II. PROCESSING FEATURES RECOGNITION METHODS BASED ON NEURAL NETWORK.

\section{E. Volume Decomposition Based Approaches}

The volume decomposition method decomposes, rearranges and reorganizes the cutting volume between the bank and the finished part, and generates characteristic interpretation. The typical method is convex shell decomposition and unit body decomposition. Wang can obtain the similarity of two three-dimensional models by constructing a shape distribution curve, which can be implemented to the matching of volume decomposition method [23].

In the detection and identification of the ring, Koo identifies the concave feature by detecting the convex inner ring [24]. Kim performs stepwise volume decomposition on the ring to identify the machining features, and can effectively identify convex features, planar rings, and single intersecting features [25]. By introducing an orthogonal boundary factor, Woo can see if there are convex features surrounded by a complete concave ring, which is instructive for how to identify convex features [26]. Bok used the machining surface geometry type and ring type to identify the concave and convex areas, and described in depth how to generate a surface machining area through a series of modeling [27].

Volume decomposition based method can better identify the general intersecting features, but the method itself does not consider the processing method of the features, so it is easy to generate a lot of useless interpretation when identifying the intersecting features. In addition, the method requires a high shape of the part, and a large number of Boolean operations are required in the decomposition and recombination of the feature, and the recognition efficiency is low.

\section{F. Manufacturing Resource Based Approaches}

The foregoing methods all use the predefined feature patterns to match and interpret the part model geometry. However, due to the limited feature pattern, the geometric shapes generated by different processing methods cannot be raised without limitation, resulting in the existence of the above methods. The characteristics of the feature cannot be identified, but the identifiable features cannot be processed in two types of problems. To solve this problem, a method of manufacturing resources mapping has been put forward. Gaines re-expressed the feature definition as a set of surfaces that were continuously machined under the same tool and the same clamping conditions [28]. Houshmand translates the process of identifying features into a process of selecting machining methods and tools [29]. Built on the manufacturing resource identification method, further summarized its naming as the processing feature identification method for manufacturing resource based method. The implementation process is illustrated in Fig. III. According to the manufacturing resource factors such as the accessibility of the tool, the path of the tool and the clamping method, Sridharan divides the machining features, summarizes the boundary representation of the machining features, and realizes the recognition of the 
machining features by searching the boundary representations

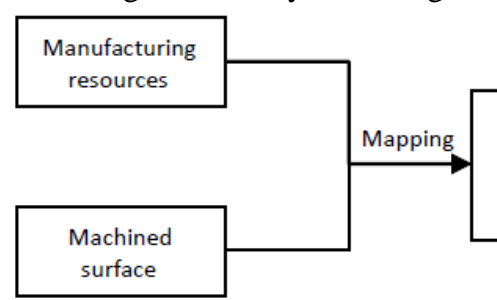

of these features [30].

\section{FIGURE III. PROCESSING SURFACE IDENTIFICATION METHOD FOR MANUFACTURING RESOURCE MAPPING}

Compared with other methods, the method of processing surface-manufacturing resource mapping has great advantages in the identification of the intersection features of complex parts. And because the actual processing and manufacturing resources in the process environment are fully considered and the processing surface processing method is optimized, the feature interpretation generated by the identifiable part features has important guiding significance for the part processing and manufacturing, and the method has strong practicability. How to more effectively and fully utilize manufacturing resource information is the key to affect the final recognition effect of the processing surface-manufacturing resource mapping, and further enhancing its recognition flexibility is another important issue that needs to be considered in the next step.

\section{G. Hybrid Approaches}

Researchers have made significant research work on hybrid methods. Rahmani proposed a hybrid recognition method based on graphs and traces, which can automatically identify intersecting features [5].

In recent years, various hybrid recognition methods are quickly developed. The processing surface-manufacturing resource method is mainly combined with the hybrid method of multiple processing feature recognition methods. It has a beneficial effect on the identification of complex box intersection features. Based on the graph matching method, considering manufacturing resource information and absorbing other the hybrid method with method advantages can identify some compound curved surfaces of aircraft structural parts and has high accuracy. The hybrid method using neural network method has higher recognition efficiency for the 3D model processing features of parts. Hybrid methods become increasingly focused on the comprehensive utilization of manufacturing resources in the process environment to enable identifiable features to guide the manufacturing of parts.

\section{COMPARISON OF 3D MODEL PROCESSING FEATURE RECOGNITION METHODS}

At present, there are many methods for identifying the processing features of 3D models, and comparing them with each other obviously has important guiding significance for future theoretical research and development of new technologies. A comparison of several methods is shown in TABLE I.

\section{TABLE I. COMPARISON OF SEVERAL 3D MODEL PROCESSING FEATURE RECOGNITION METHODS}

\begin{tabular}{|c|c|c|c|c|c|c|c|}
\hline Performance & $\begin{array}{l}\text { Rule-based } \\
\text { method }\end{array}$ & $\begin{array}{l}\text { Graph-based } \\
\text { method }\end{array}$ & $\begin{array}{l}\text { Trace based } \\
\text { method }\end{array}$ & $\begin{array}{l}\text { Neural network } \\
\text { based method }\end{array}$ & $\begin{array}{c}\text { Volume decomposition } \\
\text { based method }\end{array}$ & $\begin{array}{c}\text { Manufacturing } \\
\text { resource based } \\
\text { method }\end{array}$ & $\begin{array}{l}\text { Hybrid } \\
\text { method }\end{array}$ \\
\hline Efficiency & Medium & Medium & Medium & High & Lower & Higher & Higher \\
\hline Accuracy & Lower & High & Higher & Higher & Medium & Higher & Higher \\
\hline Flexibility & Lower & Higher & Higher & Medium & Medium & Higher & High \\
\hline Machinability & Good & Good & Good & Good & Poor & Perfect & Better \\
\hline $\begin{array}{l}\text { Manufacturing } \\
\text { resource utilization }\end{array}$ & Good & Good & Good & Good & Low & High & Higher \\
\hline
\end{tabular}

In terms of recognition efficiency, based on graphs, trace-based, and rule-based methods, subgraph matching, trade matching, and rule matching are required respectively, and the recognition efficiency is general. The volume decomposition based method requires a large number of Boolean operations, and the recognition efficiency is low. How to further compress the search space of matching information, improve the utilization of feature information, and select and improve the existing matching algorithm strategy, so as to improve the recognition efficiency of these processing feature recognition methods, further research is required. The processing method is limited, so the recognition efficiency of the method of manufacturing resources based is relatively high. The neural network method after a large number of sample training can identify the typical independent features more quickly, and the recognition efficiency is high.

In terms of recognition accuracy, the rule-based method can only identify independent features that can be fully described by rules, and the accuracy is poor. Volume decomposition based method can identify the independent features and the general intersection features more accurately, and the accuracy is moderate. Based on trace and neural network-based methods, the former can make the best use of feature information, while the latter possesses artificial intelligence with high accuracy. The method based on manufacturing resource mapping can accurately identify complex structures such as composite boxes and cavities, but the ability to identify complex free-form 
surface features needs further study. The graph-based method can better identify common features, partial intersections and surface features, and has high accuracy.

In terms of recognition flexibility, the rule-based method can effectively identify individual features, but cannot identify intersecting features, and the recognition flexibility is low. The method based on volume decomposition can identify broad intersecting features but has multiple interpretations when dealing with complex intersecting features. Flexible medium; the neural network-based method has the ability of fuzzy recognition, can identify independent, incomplete and general intersection features, and recognizes moderate flexibility; the graph-based, trace-based method utilizes geometric topological information of features, which is good Identifying common single features, and by extending and extending the attribute adjacency map, adding virtual chains, suppressing specific features, can identify general intersecting features and even partial surface features, and the recognition flexibility is higher; the method based on manufacturing resources mapping can effectively identify most intersecting features of complex parts, but it is difficult to identify complex free-form surfaces, and it is a method of recognizing high flexibility. The hybrid method optimizes and integrates a variety of methods, and the recognition flexibility is high.

In terms of processability and utilization of manufacturing resources information, the volume decomposition method uses Boolean operation to directly decompose and recombine, and some of the identified features cannot be processed, and the processability of the identifiable features is inadequate. And the use of manufacturing resource information in the process environment is neglected, and the utilization rate of manufacturing resource information is low. The rule-based, graph-based, trace-based, neural network-based approach utilizes some of the manufacturing resource information and performs well in terms of processability and utilization of manufacturing resource information. The method based on manufacturing resource mapping makes extensive use of manufacturing resource information, and has obvious advantages in processability and utilization of manufacturing resource information.

The hybrid processing feature recognition method draws on the characteristics and advantages of various methods, has good comprehensiveness, can achieve more efficient, accurate and effective identification, and has advantages in processability and utilization of manufacturing resource information.

\section{SUMMARY AND PROSPECT}

At present, processing feature recognition technology has developed rapidly, and the integration, automation degree and product process design efficiency of CAD/CAPP/CAM of modern manufacturing enterprises including this technology has been greatly improved. The existing processing feature recognition technology can well identify simple and partially complex component features, but when the part structure is more complex, contains more intersecting features and complex irregular free-form surface features, the existing processing feature recognition method still There are difficult identification and multiple interpretation problems of features, and there is still room for further improvement in recognition accuracy, recognition efficiency and recognition flexibility. Some methods have insufficient utilization of manufacturing resource information in the process environment, resulting in a large number of useless explanations or non-actual processability of some identifiable features, which seriously affect the processing and manufacturing of parts features. In addition, the partial processing feature recognition system selects or processes the complex features of the parts of a human-computer interaction manner, and the degree of automation is low.

In view of the existing problems of the existing 3D model processing feature recognition technology, the next research focus should be placed on the following aspects:

(a) Deeply do research on the processing and recognition of complex intersection features and complex space free-form surface features, improve their recognition efficiency and accuracy, and minimize human-computer interactive operation.

(b) Draw up a more flexible processing feature recognition method to further expand the range of identifiable features.

(c) To develop a new hybrid processing feature recognition technology, in particular, how to optimize and select multiple processing feature recognition methods. It is also another development of the hybrid approach to classifying features and identify them using multiple feature recognition methods.

(d) Develop a processing feature recognition technology that can more effectively utilize the manufacturing resource information of the part processing feature, so that the recognition accuracy and efficiency are improved, and the identifiable feature has processability.

(e) The processing feature recognition system needs to further have the ability to automatically mine and store the parts manufacturing resource information, to create a creative learning method for identifying the complex features of the three-dimensional model of similar parts, to verify the validity of the features and to perform the feature recognition results. Ability to automate diagnostics. The development of efficient, accurate, flexible, automatic and intelligent processing feature recognition system will grow up to be an important research topic in the field of computer integrated manufacturing in the future.

ACKNOWLEDGEMENT

Declaration of personal and funding interests: None.

\section{REFERENCES}

[1] Sendler, U. (2013). Industrie 4.0. Springer Berlin Heidelberg.

[2] S Zhang, Y Liu, X Fang (2009). Research of feature recognition method for box part based on STEP. International Conference on Measuring Technology \& Mechatronics Automation, 2009, 3(3):108-112.

[3] Ismail, N., Bakar, N. A., \& Juri, A. H. (2005). Recognition of cylindrical and conical features using edge boundary classification. International Journal of Machine Tools \& Manufacture, 45(6), 649-655.

[4] Stefano, P. D., Bianconi, F., \& Angelo, L. D. (2004). An approach for feature semantics recognition in geometric models. Computer-Aided Design, 36(10), 993-1009.

[5] Rahmani, K., \& Arezoo, B. (2007). A hybrid hint-based and graph-based framework for recognition of interacting milling features. Computers in 
Industry, 58(4), 304-312.

[6] Sadaiah, M., Yadav, D. R., Mohanram, P. V., \& Radhakrishnan, P. (2002) A generative computer-aided process planning system for prismatic components. International Journal of Advanced Manufacturing Technology, 20(10), 709-719.

[7] Sunil, V. B., \& Pande, S. S. (2009). Automatic recognition of machining features using artificial neural networks. International Journal of Advanced Manufacturing Technology, 41(9-10), 932-947.

[8] Nasr, A., Emad, S., Kamrani, \& Ali, K. (2006). A new methodology for extracting manufacturing features from cad system. Computers \& Industrial Engineering, 51(3), 389-415.

[9] Marchetta, M. G., \& Forradellas, R. Q. (2010). An artificial intelligence planning approach to manufacturing feature recognition. Computer-Aided Design, 42(3), 248-256.

[10] Zhang, K. F., Wright, A. J., \& Davies, B. J. (1989). A feature-recognition knowledge base for process planning of rotational mechanical components. International Journal of Advanced Manufacturing Technology, 4(1), 13-25.

[11] Park, M. W., \& Ko, H. (1996). A feature recognition system with interactive feature inspection and deletion capabilities. International Journal of Advanced Manufacturing Technology, 12(3), 190-196.

[12] Narang, R. V. (1996). An application-independent methodology of feature recognition with explicit representation of feature interaction. Journal of Intelligent Manufacturing, 7(6), 479-486.

[13] Joshi, Chang, \& T., C. (1988). Graph-based heuristics for recognition of machined features from a 3d solid model. Computer-Aided Design, 20(2), 58-66

[14] Li, Y. G., Ding, Y. F., Mou, W. P., \& Guo, H. (2010). Feature recognition technology for aircraft structural parts based on a holistic attribute adjacency graph. Proceedings of the Institution of Mechanical Engineers Part B Journal of Engineering Manufacture, 224(2), 271-278.

[15] Dao-Jiang, O. U., Wang, J., Sun, J., \& Shu, Q. L. (2011). Study of manufacturing features automatic recognition based on feature base-face. Modular Machine Tool \& Automatic Manufacturing Technique.

[16] Wang, J., Dao-Jiang, O. U., Shu, Q. L., \& Wang, G. X. (2014) Interacting feature recognition technology based on step-nc. Computer Integrated Manufacturing Systems, 20(5), 1051-1061.

[17] Sunil, V. B., Agarwal, R., \& Pande, S. S. (2010). An approach to recognize interacting features from b-rep cad models of prismatic machined parts using a hybrid (graph and rule based) technique.Computers in Industry, 61(7), 686-701.

[18] Gao, S., Zhao, W., Lin, H., Yang, F., \& Chen, X. (2010). Feature suppression based cad mesh model simplification. Computer-Aided Design, 42(12), 1178-1188.

[19] Da-Lei, L. I., Chen, G. F., \& Yin, Y. F. (2013). Hybrid recognition of machining features based on graph. Modular Machine Tool \& Automatic Manufacturing Technique.

[20] Li, H., Huang, Y., Sun, Y., \& Chen, L. (2015). Hint-based generic shape feature recognition from three-dimensional b-rep models. Advances in Mechanical Engineering, 7(4).

[21] Yong TaeJun, \& Raja, V. (2002). Extracting geometric attributes directly from scanned data sets for feature recognition. International Journal of Computer Integrated Manufacturing, 15(1), 50-61.

[22] Tan, C., Zhou, L., An, L., \& Zhou, R. (2005). Design and implementation of an automatic feature recognizer based on bp network in reverse engineering. Journal of Computer Aided Design \& Computer Graphics,17(10), 2305-2311.

[23] Wang, H., Zhang, S., Zhang, K., \& Bai, X. (2008). A Shape Distributions Retrieval Algorithm of 3D CAD Models Based on Normal Direction.Young Computer Scientists, 2008. Icycs 2008. the, International Conference for (Vol.15, pp.891-896). IEEE.

[24] Koo, S., \& Lee, K. (2002). Wrap-around, operation to make multi-resolution model of part and assembly. Computers \& Graphics, 26(5), 687-700.

[25] Kim, B. C., \& Mun, D. (2014). Stepwise volume decomposition for the modification of b-rep models. International Journal of Advanced Manufacturing Technology, 75(9-12), 1393-1403.

[26] Woo, Y., \& Kim, S. H. (2014). Protrusion recognition from solid model using orthogonal bounding factor. Journal of Mechanical Science \& Technology, 28(5), 1759-1764.

[27] Bok, A. Y., \& Mansor, M. S. A. (2013). Generative regular-freeform surface recognition for generating material removal volume from stock model. Computers \& Industrial Engineering, 64(1), 162-178.

[28] Gaines, D. M., \& Hayes, C. C. (1999). Mediator: a resource adaptive feature recognizer that intertwines feature extraction and manufacturing analysis. Asme Journal of Mechanical Design, 121(1), 145--158.

[29] Houshmand, M., \& Imani, D. M. (2009). A volume decomposition model to determine machining features for prismatic parts. Journal of Applied Sciences, 9(9).

[30] Sridharan, N., \& Shah, J. J. (2004). Recognition of multi axis milling features: part i-topological and geometric characteristics. Journal of Computing \& Information Science in Engineering, 4(3), 242-250. 